\title{
Optimizing Systemic Therapy Selection in Metastatic Colorectal Cancer
}

Presented by Axel Grothey, MD

\begin{abstract}
For metastatic colorectal cancer, many effective agents can be incorporated into multiple lines of therapy across the nearly 3-year continuum of treatment. Emphasis should be on using all the recommended agents, with extended duration of anti-vascular endothelial growth factor treatment, and on identifying patients with any mutation in the RAS pathway. Chemotherapy plus bevacizumab or an epidermal growth factor receptor antibody are both viable options as first-line therapy in patients with $R A S$ wild-type disease. (J Natl Compr Canc Netw 2015;13:682-685)
\end{abstract}

"Long-term survival for patients with metastatic colorectal cancer [CRC] is not based on choice of firstline therapy, but on the incremental benefits that accrue over time using available agents," according to Axel Grothey, MD, Professor of Oncology at Mayo Clinic.

Treatment of metastatic CRC in 2015 can include induction, maintenance, reintroduction of first-line agents, treatment beyond progression, treatment breaks, and additional lines of therapy (Figure 1).

"With current strategies, we have improved median overall survival to more than 30 months. It's not one breakthrough drug but the art of oncology that keeps our patients alive," Dr. Grothey said.

At the NCCN 20th Annual Conference, Dr. Grothey advised clinicians on the optimal use of "tools" to individualize treatment beyond the recommendations found in NCCN Clinical Practice Guidelines in Oncology (NCCN Guidelines) for CRC.

Presented by Axel Grothey, MD, Professor of Oncology, Mayo Clinic, Rochester, Minnesota.

Dr. Grothey has disclosed that he has received grants or research support from Bayer HealthCare, Eisai Inc, Eli Lilly and Compay, Genentech, Inc., and Pfizer Inc.

Correspondence: Axel Grothey, MD Mayo Clinic Cancer Center, 200 First Street SW, Rochester, MN 55905.

E-mail: grothey.axel@mayo.edu

\section{Treatment Paradigms, in Brief}

Dr. Grothey summarized a few key points for treating metastatic CRC:

- Some patients with stage IV disease can be cured using an interdisciplinary approach (ie, surgical resection), though the vast majority will not be.

- The goal in the palliative setting is to extend duration of life while maintaining quality of life. In this setting, FOLFOX = CapeOX = FOLFIRI.

- The addition of biologics to chemotherapy has improved outcomes, but not as much as anticipated.

- Extended inhibition of the vascular endothelial growth factor (VEGF) is important in disease control; this involves treatment until progression, maintenance therapy, and treatment beyond progression using these agents.

- Monoclonal antibodies directed against epidermal growth factor receptors (EGFRs), cetuximab and panitumumab, are interchangeable.

- Patients should be tested for all RAS mutations to identify patients who will benefit from anti-EGFR agents.

- Chemotherapy plus either bevacizumab or EGFR antibodies are both viable options as first-line therapy in patients with RAS wild-type mutations.

\section{Biologics Have Improved CRC Outcomes}

With chemotherapy alone, median overall survival (OS) never exceeded 2 years. The biologics-bevacizumab, cetuximab, panitumumab, ziv-aflibercept, and regorafenib-have increased OS to 30 months or more. It is the clinician's task to use these effective agents optimally, Dr. Grothey emphasized.

Multiple trials established the benefit of adding the anti-VEGF agent bevacizumab to chemotherapy, which 


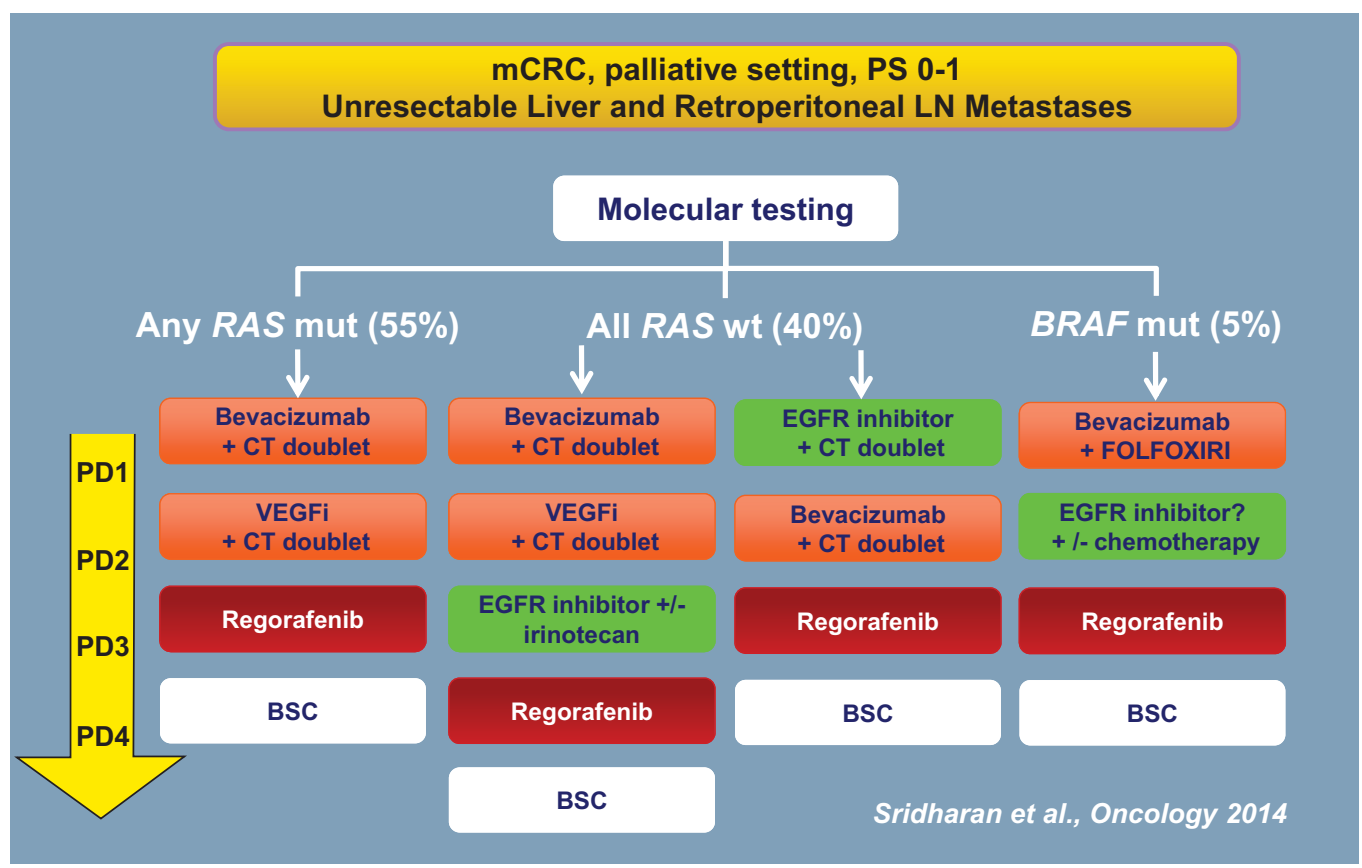

Figure 1 A classic case of metastatic colorectal cancer in 2015. Reprinted from Oncology 2014;28:110-118.

reduces the risk of progression by as much as $46 \%$. To reduce neurotoxicity associated with prolonged bevacizumab use, Dr. Grothey advised clinicians to discontinue "the toxic component of the regimen," oxaliplatin, and continue the remaining drugs, with bevacizumab, until progression. "This is how to optimize the use of first-line bevacizumab," he indicated.

Clinical synergy is observed when bevacizumab is given with a fluoropyrimidine, a first-line regimen recommended by the NCCN Guidelines. This combination proved its worth in the European AVEX study that involved elderly patients by reducing progression by $47 \%(P<.001)$ and adding 4 months median OS. ${ }^{1}$

Summing up induction therapy, Dr. Grothey advised clinicians to include oxaliplatin for 8 cycles, then to maintain response with fluoropyrimidine/ bevacizumab, "because duration of anti-VEGF therapy matters, and bevacizumab beyond progression can provide some survival benefit," he said. A regimen he recommends is low-dose capecitabine, given continuously with bevacizumab every 3 weeks.

Other VEGF inhibitors-ramucirumab (approved in gastric and non-small cell lung cancers) and ziv-aflibercept-have also been shown in phase III trials to yield a modest OS benefit, "but there are no compelling arguments for using these drugs over bevacizumab," Dr. Grothey added.

\section{Triplets Up Front}

Some patients may need more active treatment initially, and triplets can be useful in these circumstances, he continued.

The recent TRIBE study randomized 508 untreated patients to receive FOLFIRI or FOLFOXIRI, both with bevacizumab, for up to 12 cycles, followed by 5 -fluorouracil/leucovorin plus bevacizumab as maintenance until progression ${ }^{2}$ FOLFOXIRI/bevacizumab was associated with a median progression-free survival (PFS) of 12.1 months, versus 9.7 months with FOLFIRI/bevacizumab (hazard ratio [HR],0.75; $P=.003$ ), higher response rates, and a trend toward improved OS.

Dr. Grothey suggesting taking this approach in the $8 \%$ of patients with BRAF-mutated tumors. These patients typically have poor outcomes and therefore warrant more aggressive treatment. Unfortunately, the BRAF inhibitor vemurafenib has not been proven beneficial in these tumors. The phase II SWOG 1406 trial is evaluating dual BRAF and EGFR inhibition.

\section{Anti-VEGF Agents as Salvage Therapy}

For patients with refractory disease, multikinase inhibitors may be a means of "challenging the many ki- 
Grothey

nases that may be involved in secondary resistance," he said.

The multikinase inhibitor regorafenib met its primary end point in the CORRECT trial by improving OS (HR, 0.77; P=.0052) and PFS (HR, $0.49 ; P<.000001) .{ }^{3}$ These findings were supported by the Asian CONCUR study, which showed an even greater benefit in patients with no prior targeted therapy (HR, 0.31). ${ }^{4}$

HRs for regorafenib were some of the strongest observed in contemporary metastatic CRC trials, and the drug is currently being tested in earlier lines of treatment. However, off-label use is not recommended.

On the horizon for salvage therapy is a novel oral nucleoside agent, TAS-102, which combines trifluorothymidine and tipiracil hydrochloride. In the RECOURSE study, an OS benefit was seen versus best supportive care $(\mathrm{HR}, 0.68 ; \mathrm{P}<.0001){ }^{5}$

"This is a drug that can delay tumor progression, with fairly mild toxicity, in distinction to regorafenib," Dr. Grothey pointed out. "It will be interesting to see how we will sequence these 2 drugs when TAS-102 is on the market."

\section{Testing for RAS Mutations}

Multiple studies have confirmed the benefit of EGFR antibodies in patients lacking RAS mutations. The importance of patient selection was clear from the PRIME study, which not only proved the OS benefit of panitumumab plus FOLFOX4 in patients with KRAS wild-type mutations, but showed a $72 \%$ increased risk of progression when the EGFR inhibitor was given to patients with mutant KRAS. ${ }^{6}$

Further RAS testing of the population in the PRIME study revealed the importance of additional mutations in KRAS and NRAS, which were identified in $17 \%$ of patients with nonmutated KRAS exon 2. These mutations were also associated with inferior PFS (HR, 1.28) and OS (HR, 1.29) with panitumumab.

Because 1 of 6 patients with wild-type KRAS has other RAS mutations, the NCCN Guidelines for Colorectal Cancer recommend comprehensive RAS testing.

\section{Which Targeted Treatment, First?}

The choice of first-line targeted therapy continues to be debated, though the recommended agents are viewed as equivalent.

In FIRE-3, cetuximab given with FOLFIRI did not significantly increase response rate over bevaci-

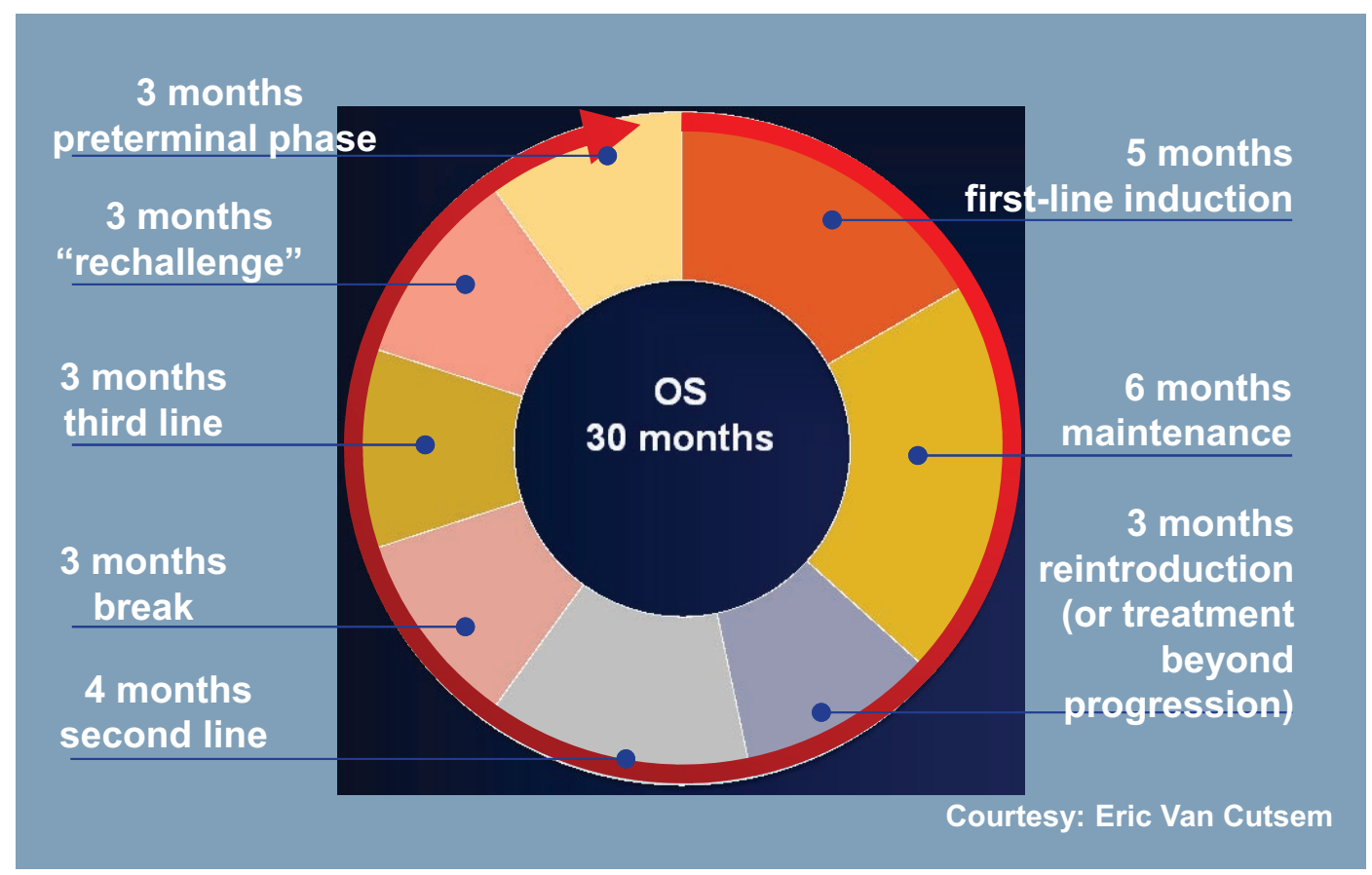

Figure 2 One possible optimized treatment strategy. 
zumab/FOLFIRI (primary end point) and did not improve PFS, but did improve OS (HR, 0.77; $P=.017$ ), though the 3.7-month improvement "was driven by only 13 patients," Dr. Grothey pointed out.

In the all-RAS population, the OS difference was more pronounced-7.5 months (HR, 0.70; $P=.011$ ), and the response rate differences became statistically significant. ${ }^{8}$

The findings might be explained by imbalances in postprogression therapy, an effect of first-line therapy on the sensitivity of cancer cells to subsequent treatment, and a positive effect on a subpopulation of patients who maintain long-lasting treatment benefit, Dr. Grothey proposed.

A larger confirmatory trial with a more appropriate primary end point was needed, and this was provided by CALGB/SWOG 80405. ${ }^{9}$ No differences in PFS or OS were noted between cetuximab and bevacizumab (plus FOLFOX or FOLFIRI), with median OS approximately 30 months in each arm. Additional analysis of all-RAS wild-type patients did not alter outcomes.

Dr. Grothey maintained that these 2 important trials are more similar than different because they showed consistent trends in response rates, PFS, and OS.

Considering that several first-line options are equivalent, Dr. Grothey proposed that the molecular profile can guide initial treatment, but multiple strategies are acceptable (Figure 2).

\section{References}

1. Cunningham D, Lang I, Marcuello E, et al. Bevacizumab plus capecitabine versus capecitabine alone in elderly patients with previously untreated metastatic colorectal cancer (AVEX): an open-label, randomised phase 3 trial. Lancet Oncol 2013;14:1077-1185.

2. Loupakis F, Cremolini C, Masi G, et al. Initial therapy with FOLFOXIRI and bevacizumab for metastatic colorectal cancer. $\mathrm{N}$ Engl J Med 2014;371:1609-1618.

3. Grothey A, Van Cutsem E, Sobrero A, et al. Regorafenib monotherapy for previously treated metastatic colorectal cancer (CORRECT): an international, multicentre, randomised, placebo-controlled, phase 3 trial. Lancet 2013;381:303-312.

4. Kim $\mathrm{T}, \mathrm{Xu}$ R, Yau $\mathrm{T}$, et al. CONCUR: a randomized, placebo-controlled phase 3 study of regorafenib (REG) monotherapy in Asian patients with previously treated metastatic colorectal cancer (mCRC) [abstract]. Presented at the 2014 ESMO Congress; September 26-30, 2014; Madrid, Spain. Abstract 5000.

5. Yoshino T, Mayer R, Falcone A, et al. Results of a multicenter, randomized, double-blind, phase III study of TAS-102 vs placebo, with best supportive care in patients with metastatic colorectal cancer refractory to standard therapies [abstract]. Ann Oncol 2104;25(Suppl 2):114.

6. Douillard J-Y, Oliner KS, Siena S, et al: Panitumumab-FOLFOX4 treatment and RAS mutations in colorectal cancer. N Engl J Med 2013;369:1023-1034.

7. Heinemann V, von Weikersthal LF, Decker T, et al. FOLFIRI plus cetuximab versus FOLFIRI plus bevacizumab as first-line treatment for patients with metastatic colorectal cancer (FIRE-3): a randomised, openlabel, phase 3 trial. Lancet Oncol 2014;15:1065-1075.

8. Stintzing S, Jung A, Rossius L, et al. Analysis of KRAS/NRAS and BRAF mutations in FIRE-3: a randomized phase III study of FOLFIRI plus cetuximab or bevacizumab as first-line treatment for wild-type KRAS (exon 2) metastatic colorectal cancer patients [abstract]. Eur J Cancer 2013;49(Suppl 3):Abstract 17.

9. Venook AP, Niedzwiecki D, Lenz HJ, et al. CALGB/SWOG 80405: phase III trial of irinotecan/5-FU/leucovorin (FOLFIRI) or oxaliplatin/5-FU/ leucovorin (mFOLFOX6) with bevacizumab or cetuximab for patients with KRAS wild-type untreated metastatic adenocarcinoma of the colon or rectum [abstract]. J Clin Oncol 2014;32(Suppl 5):Abstract LBA3. 